\title{
The Study on Digital Traffic Service System and Key Technologies of Location Based Service
}

\author{
TANG Liwen \\ The Academy of Equipment \\ 75,P.O.Box 3380 Beijing 101416 P.R. China \\ tangli_wen@sina.com
}

\author{
YUWEN Jingbo \\ The Academy of Equipment \\ Beijing, P.R. China
}

\begin{abstract}
The system of location based service has become more popular in the applications of digital traffic. The situation of vehicle navigation and location based service is introduced. The functions of one digital traffic system of location based service are proposed from three aspects about preparation and pretreatment of map data, intelligent voice hint of vehicle navigation and advertisement service based on location. Then the system which includes software and hardware structures is designed. Among the structures, the hardware includes GPS receiver and vehicle device based on WinCE, while the software is composed by two parts, one is the map data preparation and pretreatment software, and the other is the intelligent voice hint navigation software of vehicle PC. Lastly the key technologies of map display, map matching, intelligent path planning and voice navigation are explored in detail. The result can provide the technique support and reference for developing city traffic service system.
\end{abstract}

Index Terms-location based service; digital traffic; vehicle navigation; path planning

\section{INTRODUCTION}

\section{A. Vehicle Navigation Technology}

Since the Global Position System (GPS) officially goes into use, the most active domains about GPS applications in global are military surveying and mapping, precision measurement, navigation position and traffic management, geological study, and etc. The flexible use of vehicle navigation technology has provided the widespread and deeply helps for modern convenient traffic. By receiving the signals of satellite, the vehicle navigation system can provide such functions as vehicle navigation, optimal selection of traveling route, information query of position, and correction of path deviation for drivers. All of these functions are realized by coordinating with their stored electrical map and inertial navigation devices of GPS and gyroscope. Nowadays, the development of vehicle application is divided into two types [1,2], one is vehicle tracking which is used for anti-theft, the other is vehicle navigation system which is used for autonomous navigation. One big characteristic of GPS receiving system is only signal receiving and without sending. So the GPS tracking system for anti-theft gives the corresponding solution by communication network and GPS vehicle device of anti-theft. The vehicle navigation device can master its direction and destination properly by receiving signals of satellite and coordinating with electrical map.

The exploitation and application of vehicle navigation technologies are always the pot of study in most high technology companies, universities and colleges. They have played an important role in creativity of traffic system by integrating the domain of vehicles, traffic, computer, geographic information, communication and system science. Now, the most advanced places of these technologies are American, Japan and Europe where their present situation represents the main developing orientation of this domain. The GPS vehicle navigation system of our country is also developing to the integration of information terminals, realtime of traffic information, platform-based information service and networked data update [3].

\section{B. Application of Location Based Service}

The location based service (LBS) is one valued-added service which can provide the corresponding service for users by using certain kind of technology means. This service is supported by the platform of electrical map, and can get the position of longitude and latitude about mobile terminal users by communication network [4]. It includes two meanings, one is to determine the geographic position of mobile device and users, the other is to provide all kinds of services relating to position. This is also defined as position service or mobile position service. For example, first it finds the geographic position of current user, and then queries the name and address of guesthouse, cinema, library and gas station from current position to $1 \mathrm{~km}$ in certain map range. So using network and wireless network, the LBS can finish two functions of position and service between fixed users and mobile users. Such as GPS position searching of CARELAND navigation and on line position of Google map.

There are two kinds of applications about position: one is cooperation which binds the position and manufacturer. So all of these are user experience applications which grow up with users when finding whether coffee hall or gymnasium, resolving whether dinner or leisure. This kind has better effects. The second application is to solve path status, parking, oil fee and dynamic route navigation [5]. And the extent application can broadcast in real-time through the power function of voice, 
especially in some enormous population cities. Thus, the voice advertisement can be appropriate.

\section{SYSTEM FUNCTION}

The system mainly includes the functions about the preparation and pretreatment of map data, intelligent voice hint of vehicle navigation, advertisement service of television video and advertisement play based on position.

\section{A. Preparation and Pretreatment of Map Data}

1) Data Edition: It corrects the existing errors of the gathered data and modifies the geometrical data and attribute code through the addition, deletion and modification to the map elements. The edition is realized by two methods, one is to correct the obvious illogical data by itself judgment and check to the program; the other one is to modify the data in the graphical interfaces by the type of human-computer interaction.

2) Data Compression: In order to decrease the storage number of data, save the storage space, increase the speed of display and analysis, the origin data must be compressed. The data compression mainly includes deleting the redundant data from the origin one, and transforms from the real coordinate to the corresponding coordinate with lower storage.

3) Data recombination: According to the characteristics of slow processing speed about the embedded system CPU, few storage of peripheral, and small display screen, the data needs certain recombination that includes layered, splitting blocks and established index to meet the vehicle navigation.

4) Topology Data Creation: The most key elements are to analyze the optimal path in vehicle navigation system, then the topology relationship of the route date must be created.

5) Data Output: It mainly finishes the function of transforming the treated data to the format of the system needed.

\section{B. Intelligent Voice Hint of Vehicle Navigation}

1) Map Display: The map has the mode of day and night display background, navigation mode of rolling and normal, local zoom in mode of crossed partial route with double frame. The normal mode is to keep the orientation of the electrical map to north all the time during the navigation. The rolling mode is to keep the traveling orientation consistent when the electrical map rolls which is caused by the change of traveling orientation. For the sake of guidance and query convenience, this function can also provide different views about scale.

2) Navigation Function: The function includes the receiving and parsing of GPS signals, display of satellite ephemeris and status, time of GPS, direction of vehicle traveling and display of vehicle position in real-time. It can also fix the real-time position of vehicle which the actual position declination should be less than $20 \mathrm{~m}$. The manner of navigation is to provide such functions as clear voice and visual map for dual guidance, traveling direction of intuitionistic name, distance to the destination and next inflexion distance of exact calculation, observable crossing or crossroads and high-speed passageway of graphic show. If the vehicle departures from the appointed route, it will turn to the right one by guiding the new route automatically and quickly.

3) Information Query: The function will offer many kinds of query types, such as address query, interest place query, road name query, circumjacent establishment query and etc.

4) Path Planning: According to the current position and destination position of vehicle, it can plan path intelligently and recalculate automatically after departing from the schedule one under the restriction of current traffic status.

5) Voice Guidance Control: It can accomplishes the voice control and service function. Voice navigation may timely remind users to act when the vehicle travels to the crossing under the guidance route. Based on the planned route, it can guide users to the destination under the voice hint of vehicle current traveling position. For example, when the vehicles meet the crossing or swerve, the vehicle GPS voice system will clew users to turn so that they can avoid detour. It can offer the global voice hint for users, and in this way, without looking the interface the drive will drive safely and cosily during the whole navigation.

6) Receiving and Display of Traffic Information: It can receive the data of traffic status from control center, and parse these data according to the region the vehicle positioned. After that, using words, voice and graphics types, it assists the dynamic path planning by offering the traffic management information.

7) Alert Control: Alert signal will send to the control center through network to report the exceptional information of vehicles.

\section{Advertisement Service Based on Location}

Network advertisement is always the most payoff types in the internet industry. Most burgeoning websites take more accurate, more effective, cheaper advertisement as their target. In order to avoid users' antipathy and protect their internet advertisement from junk information, the websites must be restricted strictly to their display time, places and numbers. The types of advertisement include searching text link ones in certain region, picture ones when reaching some region and interactive ones with users.

During one vehicle television program, the commercial advertisement can inter cut in one minute for every two to three minutes. This can contact with the users that traditional television advertisement cannot, such as senior leader in companies, experts which have no time to watch traditional television program in every domains. In the time riding, the special television program and advertisement environment strengthen the continuity that people take the information from $\mathrm{TV}$, and can make people subconsciously accept the advertisement and leave them the profound impression. The contents are as followed:

1) TV Videos: This is mostly the vehicle DVD function. And it can receive the digital video signal so that switch between the software and hardware in the navigation terminals.

2) Advertisement Play: The function mainly receives and stores the advertisement information through wireless, and 
plays them on the navigation map by the type of pictures, texts and etc.

\section{DESIGN OF SYSTEM}

\section{A. Vehicle Hardware Component}

Vehicle hardware is composed of the following parts:

1) GPS Receiver: The main demand is to known the position of vehicle at anytime during the navigation, and it can be used to verify the optimal route to the destination. Thus the position of vehicle can be confirmed by receiving the GPS signal through GPS receiver.

2) Vehicle Device Based on WinCE: This is the kernel component in vehicle navigation which is used for displaying video and image information of the position status. It can be selected from LCD, CRT and TV. Most of the functions about vehicle navigation can be realized based on it. For example, it can calculate the longitude, latitude, altitude, speed and time about the vehicle position.

Because of the particularity of using environment, the vehicle PC as the core of the system must have little size, high integration, low cost, power disposal, simple and convenient operation. Now most of the vehicle PCs use such embedded operation systems as WinCE and embedded Linux. According to using frequency and road complexity of vehicles, the PC must have high reliability, good expansibility and compatibility.

\section{B. Software Structure}

The software is composed by two parts, one is the map data preparation and pretreatment software, the other is the intelligent voice hint navigation software of vehicle PC. As figure 1 shows.

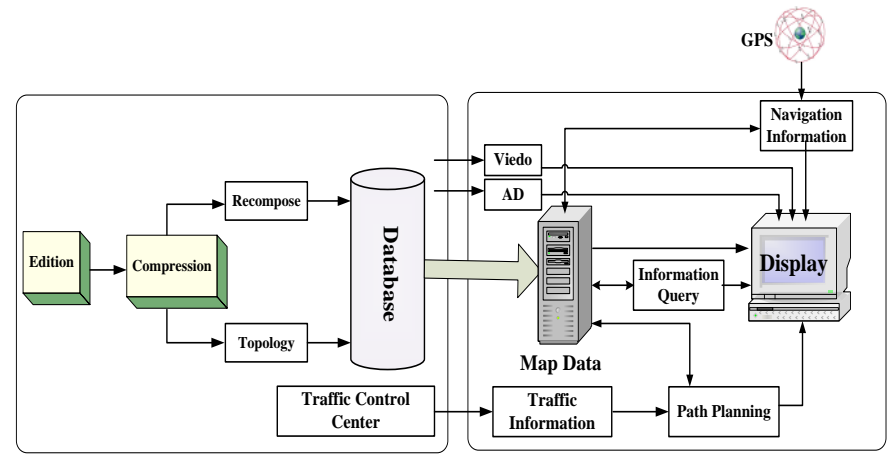

Fig. 1. Software structure of the system

1) Data Preparation and Pretreatment Software: For the convenient calculation and display, the data must be transferred to the format needed by system through a series of pretreatment.

2) Intelligent Voice Hint Navigation Software of Vehicle $P C$ : After considering synthetically the factors of running speed, display function, device cost and size about the vehicle navigation system, the paper selects Windows $\mathrm{CE}$ as the operation system. This operation system is a compact, efficient and scalable one that specially aims at the development of embedded products. The characteristics of multithreading, multitask and safely preemptive are designed specially for hardware that has restrict resources. The paper selects Microsoft Embedded Visual $\mathrm{C}++$ as the tool for software exploitation.

\section{KEY TECHNOLOGIES}

\section{A. Display of Map}

Display of map is one of the key technologies. The speed of display will directly influence the practicality of the whole system. Due to the low treatment and little memory of CPU that the embedded hardware device possessed, the system must put forward the high demand for the drawing efficiency of map. The technologies are as followed:

1) Block organization to the map data: That is to make the loading data scale finite every time, and find the balance point between the times of reading and data quantity.

2) Take layer structure as the type to organize and store data for decreasing data redundancy: During the map zoom in and out, the speed will become slow if it displays as the fixed factors, and the map cannot be read because of the high loading of screen. So the spatial data must be accepted or rejected and classified according to different scales, organized and displayed by level of model(LOD). The smaller the scale is, the display level of data is higher. And the bigger the scale is, the display of spatial data is more detailed.

\section{B. Map Matching}

1) Error of Navigation Position and Map Matching: The error that occurred in navigation position comes from two aspects, GPS error and map data error. Map data error comes from data collection error, and it can be considered as accurate data when it has high quality. It also supposes that the vehicles run on the road. So based on this hypothesis, map matching can correct the receiving GPS data based on map data, and make GPS data meet the navigation demand.

2) Map Matching of Topology Restriction: The general map matching only uses spatial position as the matching condition to find the nearest road. In this way, it will bring ambiguity of matching when the position of GPS close to the crossing node, and cannot give the right guidance. The paper plans to import the vehicle direction and topology restricted condition when carrying road matching so as to enhance the precision and speed of matching. The basic steps are: (1)Getting the origin position and vehicle direction; (2)Finding several roads nearest to the vehicle position; (3)Calculating the direction of the nearest roads according to vehicle direction, then verifying the exact road of vehicle based on the nearest principle of distance and direction; (4)Calling in topology relation, and querying the joint road with itself, then as the corresponding matching road, taking it as matching subset with current section; (5)Supposing the driving is continuous and receiving the GPS signal continuously without break. So the next data position received must be lie in current section or corresponding one, then it will match in the subset based on 
angle and position. The GPS data will be considered as invalid if the matching fails.

\section{Intelligent Path Planning}

Intelligent path planning is the core of vehicle navigation system, and becomes the key whether the system is practical or not. Intelligent path planning is to find the optimal path in the path topology net under the condition of known start point and end point. The optimal path has the nearest distance, shortest riding and least cost.

The path network must be built before analysis of the optimal path. Because of slow processing speed of vehicle PC CPU, and few storage space of peripheral, the basic demand of designing path network not only meets the optimal path requirement for calculation but also occupies the least storage space.

The classical expression method to path network is weighted directed graph. Through this method, the problem that confirms the optimal driving path between certain places can be transferred to the problem that seeks the shortest path between two points on the weighted directed graph. There are two key problems waiting for solving. One is how to define path weight, the other is what structure can be used to save path network. The weights for the path is based on the length of path, and other properties of path are translated into the length of path; Forward star structure is used for the storage structure of path network, this structure has the characteristics of small occupying storage space and fast query speed. At the same time, the traffic information is transferred into path weight to aid path planning.

\section{Voice Navigation}

The thought of voice navigation is follows:

a) Based on the result of path planning, the path series are combined with the same names.

b) The coordinate of inflexions between two adjacent paths is calculated from the result of planning. Then according to the direction of these two paths, the round condition of the inflexions is brought out, and thus a series of inflexions and their corresponding turning series are gained.

c) The GPS frame data of vehicle current position and driving direction is received. After that, the data is separated to get the vehicle current position and driving direction.

d) Map matching will be carried through based on vehicle current position and driving direction. Then the judgment will be given about whether current path is consistent with beforehand path planning. If current path differs from the planned one, voice alert hint of departure will brought out, and if the departure is large, the planning will be done again. e) If current path is consistent with one of planned path, the distance to next inflexions will be calculated. The judgment whether the voice hint in the inflexions is given will also be put forward. If the situation is satisfied, the hint will be proposed.

The three to five steps are repeated in turn until the destination or certain area of target is reached. And the access to the target is also given.

\section{CONCLUSION}

In recent years, with the widely development of GIS technology and satellite navigation technology, the intelligent traffic system to solve city traffic problems has developed more quickly. And as the most important part of intelligent traffic system, vehicle navigation system is also become one of the hot directions in current society studies.

The digital traffic service system the paper studied is location based service, and it can receive all kinds of real time traffic information from the control center through vehicle communication devices. By disposing the information combined with electrical map, the result can guide drivers to avoid traffic jam and help them to select the most optimal path in real time, so that the drivers can reach the destination in the fastest speed. Meanwhile, the advertisement service function based on position service is also can provide advertisement of text and picture for passengers. The usage of the system can insure the vehicles punctual, fast, safely and run orderly in city path network, thus the target is achieved by fully using the city path network in balanced and enhancing the efficacy of traffic transportation.

\section{REFERENCES}

[1] CHEN Tao. The Algorithms Design and Realization of Path Planning for Vehicle Navigation in Big Districts[D]. PLA Information Engineering University. 2005,4.

[2] GONG Pibo. The Study on the Intelligent in-Vehivle Navigation System[D]. PLA Information Engineering University. 2005,4.

[3] ZHU Baoshan, SHI Hui, WANG Weihong. The Software and Characteristics of Homemade Vehicle Navigation[J]. Military Surveying and Mapping. 2012, 3.

[4] Yi Jiong, ZHANG Lei, WANG Jiangyu. The Location Services Analyse and Application Research Based on Satellite Positioning[J]. WORLD SCI-TECH R\&D. 2008, Vol.30, No.3. 328-330.

[5] WU Sheng, CHEN Nan. Study on Dynamic Vehicle Routing Problem Based on Location-Based Services[J]. Journal of Fuzhou University(Natural Science). 2007, Vol.35 No.6. 953956. 